University of Nebraska - Lincoln

DigitalCommons@University of Nebraska - Lincoln

$12-22-2000$

\title{
Nonlinear effects of physisorption on static friction
}

\author{
G.T. Gao \\ University of Nebraska-Lincoln \\ Xiao Cheng Zeng \\ University of Nebraska-Lincoln, xzeng1@unl.edu \\ Dennis J. Diestler \\ University of Nebraska-Lincoln, ddiestler1@unl.edu
}

Follow this and additional works at: https://digitalcommons.unl.edu/chemzeng

Part of the Chemistry Commons

Gao, G.T.; Zeng, Xiao Cheng; and Diestler, Dennis J., "Nonlinear effects of physisorption on static friction" (2000). Xiao Cheng Zeng Publications. 49.

https://digitalcommons.unl.edu/chemzeng/49

This Article is brought to you for free and open access by the Published Research - Department of Chemistry at DigitalCommons@University of Nebraska - Lincoln. It has been accepted for inclusion in Xiao Cheng Zeng Publications by an authorized administrator of DigitalCommons@University of Nebraska - Lincoln. 


\title{
Nonlinear effects of physisorption on static friction
}

\author{
G. T. Gao and X. C. Zeng \\ Department of Chemistry, University of Nebraska-Lincoln, Lincoln, Nebraska 68588 \\ D. J. Diestler \\ Department of Agronomy, University of Nebraska-Lincoln, Lincoln, Nebraska 68583
}

(Received 1 August 2000; accepted 26 September 2000)

\begin{abstract}
The effects of a physisorbed film on the force of static friction in a model contact (monatomic adsorbate confined between plane-parallel walls) were investigated by Monte Carlo simulation. At fixed coverage the friction curve (shear yield stress vs normal stress) exhibits a marked nonlinearity, which results from a competition between adsorbate-wall interactions that predominate at low loads and wall-wall interactions that set in beyond a threshold load, which increases with coverage. Previous proximal-probe and computer experiments, carried out at high coverages, see only the initial (low-load) linear portion of the friction curve. (c) 2000 American Institute of Physics. [S0021-9606(00)70148-6]
\end{abstract}

\section{INTRODUCTION}

The classical law of friction between macroscopic objects, which states that the force of static friction is proportional to the force pressing the objects together (load), lacks a satisfactory fundamental explanation. When macroscopic surfaces are pressed together, they make molecular contact at only a few discrete asperities, the total area of which is a small fraction of the apparent interfacial area. The force of static friction (i.e., the force required to initiate sliding) is then equal to the sum over all asperities of the shear yield stress $\left(\tau_{s}\right)$ times the area of molecular contact $(A)$ of the asperity. In general, both $A$ and $\tau_{s}$ vary from asperity to asperity. To achieve a molecular understanding of friction it is desirable to study single, well characterized asperities. This is being done by means of proximal surface probes ${ }^{1}$ such as the surface forces apparatus (SFA) $)^{2,3}$ and the atomic force microscope $(\mathrm{AFM}),{ }^{4}$ as well as by analytical ${ }^{5}$ and numerical $^{6}$ treatments. The purpose of this article is to present the results of computer simulations of a model asperity that demonstrate how physisorption at the interface can give rise to a striking nonlinear dependence of $\tau_{s}$ on the load.

The force of friction $F_{s}$ on a single asperity can be expressed generally as

$$
F_{s}=\iint_{A} d \boldsymbol{R} \tau_{s}(\boldsymbol{R}),
$$

where $\boldsymbol{R}$ denotes the (two-dimensional) vector position of the element of area and the integration is over the area of molecular contact. Both $A$ and $\tau_{s}$ are implicit functions of the load $L$. If the contact is assumed to be homogeneous over $A$, Eq. (1) reduces to

$$
F_{s}=\tau_{s} A,
$$

where $\tau_{s}$ is now to be interpreted as the mean shear yield stress. Equation (2) is the usual starting point for the analysis of proximal-probe measurements. ${ }^{7}$ Both $F_{s}$ and $A$ can be measured as functions of $L$. Then $\tau_{s}$ can be calculated from
Eq. (2). Now if $\tau_{s}$ is linear in the normal stress (i.e., the stress applied perpendicularly to the interface) $\tau_{n}=L / A$, then

$$
\tau_{s}=\tau_{s, 0}+\lambda \tau_{n},
$$

and it follows from Eqs. (2) and (3) that

$$
F_{s}=\tau_{s, 0} A+\lambda L .
$$

Some SFA data, for example those on Langmuir-Blodgett monolayers $^{8}$ and on multilayer hydrocarbon films ${ }^{9,10}$ between atomically smooth mica sheets, conform to Eq. (4) as do AFM data on the sliding of an Si tip over the (001) face of GeS partly covered by patches of $\mathrm{C}_{60} \cdot{ }^{11}$

In contrast to proximal-probe experiments, computer simulations are performed on model asperities at constant area of molecular contact. They are concerned essentially with the determination of $\tau_{s}$ for homogeneous contacts. Simulations of clean crystalline surfaces sliding over each other, such as the (111) face of a $\mathrm{Cu}$ tip on the (111) face of $\mathrm{Cu}$ substrate, ${ }^{12}$ and (111) diamond surfaces terminated with $\mathrm{H}$ or alkyl radicals, ${ }^{13}$ show that $\tau_{s}$ may be a nonlinear function of $\tau_{n}$, depending on the structure of the surfaces and the relative crystallographic direction of sliding. In a recent computational study of a bead-spring model of hydrocarbons adsorbed between walls comprising incommensurate face centered cubic (fcc) (111) planes, He et al. ${ }^{14}$ found that Eq. (3) is well satisfied for a wide range of model parameters. Previous simulations ${ }^{15-17}$ of confined thin films demonstrated the strong influence of adsorbed layers on friction, but none, except that of He et al., systematically explored the dependence of shear yield stress on normal stress. Moreover, all prior simulations were performed at coverages so high or loads so low that interactions between atoms in opposite walls (wall-wall or tip-substrate interactions) were negligible. Here we present the results of a Monte Carlo study of a model contact that show how a coverage-dependent competition between wall-wall and adsorbate-wall contributions to the shear stress can lead to strong nonlinearities in the "friction curve" (i.e., $\tau_{s}$ vs $\tau_{n}$ ). 


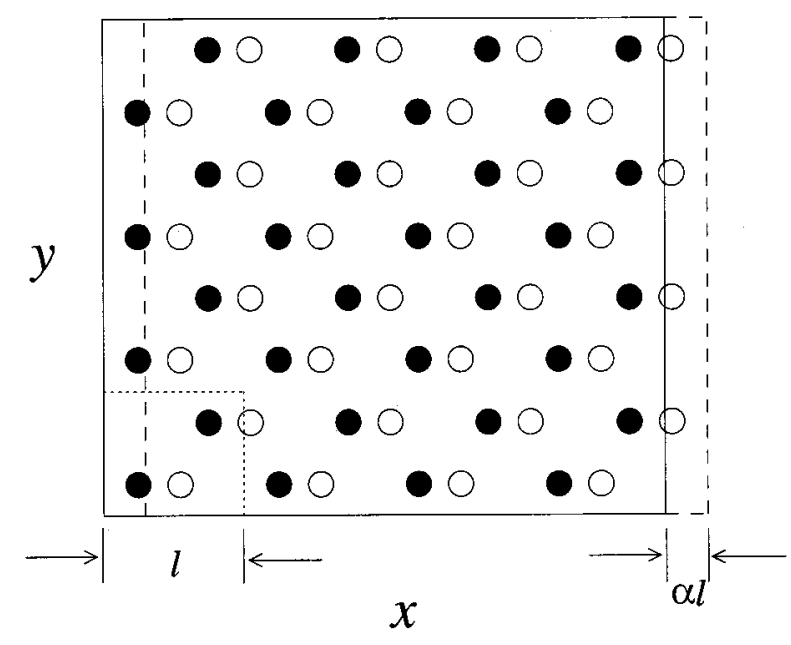

FIG. 1. Top view of system, not showing adsorbate atoms. Filled circles denote atoms in fixed wall, open circles atoms in moved wall. Small dotted square indicates unit cell of wall. Number of cells along an edge is $n=4$.

\section{MODEL}

The idealized contact, schematized in Fig. 1, comprises two identical plane-parallel walls, one of which (labeled 1) plays the role of substrate and the other of which (labeled 2) functions as the probe tip and can be translated. Each wall consists of a single fcc (100) plane containing $N_{w}=2 n^{2}$ atoms, where $n$ is the number of $l \times l$ square unit cells along an edge. The surface density of wall atoms is therefore $2 / l^{2}$. We take the walls to be rigid and to remain crystallographically aligned as wall 2 is translated. The lateral displacement of wall 2 is measured in terms of the $x$-registry $\alpha$ by the relation $\alpha l$. Thus, $\alpha$ is the fraction of $l$ by which the walls are misaligned in the $x$-direction. For simplicity we fix the $y$-registry. Between the walls are constrained $N$ atoms of monatomic film. The potential energy is taken to be a sum of Lennard-Jones $(12,6)$ interatomic potentials $u\left(r_{i j}\right)$ $=\epsilon_{i j}\left[\left(\sigma_{i j} / r_{i j}\right)^{12}-\left(\sigma_{i j} / r_{i j}\right)^{6}\right]$, where $i$ and $j$ label atoms. Since the effects with which we are concerned depend little on physically reasonable variations of the well depth $\epsilon_{i j}$, we set $\epsilon_{i j}=\epsilon$ for all pairs $(i j)$, regardless of the identities of the atoms. If $i$ and $j$ both refer either to film atoms or to wall atoms, then $\sigma_{i j}=\sigma_{F F}$ or $\sigma_{i j}=\sigma_{W W}$, respectively. If $i$ refers to a film atom and $j$ to a wall atom, or vice versa, then $\sigma_{i j}$ $=\sigma_{F W}=\left(\sigma_{F F}+\sigma_{W W}\right) / 2$. Numerical values of all quantities are henceforth given in reduced dimensionless units, in which distance is expressed in units of $\sigma_{W W}$ and energy in units of $\epsilon$. To minimize the effects of edges, we apply the usual periodic boundary conditions to the simulation cell (a square prism) in the directions of the unit-cell axes, which we take to be parallel with the $x$ - and $y$-axes of the "laboratory"' Cartesian coordinate frame (see Fig. 1).

\section{COMPUTATIONAL METHODOLOGY}

Since the shear strain rate in surface probes is typically on the order of $10^{-8} \mathrm{ps}^{-1}$, that is, extremely slow on the molecular time scale, we assume that the adsorbate remains in thermodynamic equilibrium at all instants and treat shear- ing as a quasistatic process. We compute the equilibrium properties of the system by means of the isothermalisostress Monte Carlo method, ${ }^{18}$ in which temperature $(T)$, $N, A=n^{2} l^{2}, x$-registry $(\alpha)$, and $\tau_{n}\left(=-T_{z z}\right.$, the normal stress) are controlled thermodynamic state variables. The shear stress $T_{z x}$ is given in terms of the Gibbs energy $G$ by $T_{z x}=A^{-1}(\partial G / \partial(\alpha l))_{T, N, T_{z z}} ; A T_{z x}$ is just the negative of the $x$-component of the mean force acting on the moved wall. Since the potential energy is separable into contributions due to film-film, film-wall, and (opposing) wall-wall interactions, the force acting on the walls (or the stress applied to the walls) can be expressed as a sum of film-wall and wallwall components, that is $T_{z x}=T_{z x, F W}+T_{z x, W W}$. By symmetry $T_{z x}$ is periodic in $\alpha$ with a period of unity. We take $\tau_{s}$ to be the maximum magnitude of $T_{z x}$ in the range $0<\alpha<1$. This is the minimum force that would need to be applied in order to initiate (irreversible) sliding.

\section{RESULTS AND DISCUSSION}

In all cases we set $T=1.00, n=16, N_{w}=512$, and $l$ $=1.5985$. We define the coverage by $\theta=N / N_{w}=N / 512$. Figure 2 displays plots of $T_{z x}$ and the mean separation $h$ between the walls as functions of $\alpha$ for the case $\sigma_{F F} / \sigma_{W W}$ $=1$ and $\theta=0.0195$. Since symmetry dictates that $h$ and $T_{z x}$ are, respectively, symmetric and antisymmetric about $\alpha=0$ and $\alpha=0.5$ and that both film-wall $\left(T_{z x, F W}\right)$ and wall-wall ( $\left.T_{z x, W W}\right)$ contributions to $T_{z x}$ vanish at $\alpha=0$ and $\alpha=0.5$, we need to plot these functions only on the interval $(0,0.5)$. We consider first the case of the lighter load [Fig. 2(a)], for which $T_{z x, F W}$ is positive and $T_{z x, W W}$ is negative, except over the range $0.3<\alpha<0.5$. Hence, as the walls are moved out of alignment (that is as $\alpha$ deviates from 0 ), the film-wall component of the force tends to restore them to alignment, whereas the wall-wall component tends to push them further out of alignment. Because $\left|T_{z x, F W}\right|>\left|T_{z x, W W}\right|$ for all $\alpha$, a net-restoring force tends to keep the walls aligned. In the absence of an applied shear force the walls come to rest at integral registries $(\alpha=0, \pm n, n$ an integer) where the system is stable (i.e., $G$ is minimum). By similar reasoning, the system is unstable at the half-integral registries $\alpha= \pm n / 2$ ( $n$ an odd integer). When the walls initially at rest at $\alpha= \pm n / 2$ are displaced slightly, a net force tends to push them to the next stable registry $[\alpha= \pm(n \pm 1) / 2]$. As the walls are slid reversibly out of alignment, say as $\alpha$ is increased from $0, T_{z x}$ reaches a maximum around $\alpha_{\max } \simeq 0.2$. The value of $T_{z x}$ at $\alpha_{\max }$, about 3.7, is therefore the shear yield stress $\tau_{s}$ at $\tau_{n}$ $=1.0$ [this is plotted in Fig. 3 (inset) as an open triangle].

The quantity $A \tau_{s}$ is the $x$-component of the force that must be applied to initiate (irreversible) sliding. The work required to slide (reversibly) the walls from $\alpha=0$ to $\alpha$ $=\alpha_{\max }$ is $\Delta G=A l \int_{0}^{\alpha_{\max }} T_{z x}(\alpha) d \alpha>0$. As the walls slide over this range, they also move slightly closer together, as indicated by the plot of $h$ vs $\alpha$ in Fig. 2(a). When the walls are aligned $(\alpha=0)$, the adsorbate atoms fit well into the cavities created in concert by the atoms in the two walls. Indeed, we can view the adsorbate as a fragment of the solid fcc (100) monolayer that would be present were $\theta=1.0000 .{ }^{15}$ The (positive) area under the plot of $T_{z x, F W}$ is proportional to the 

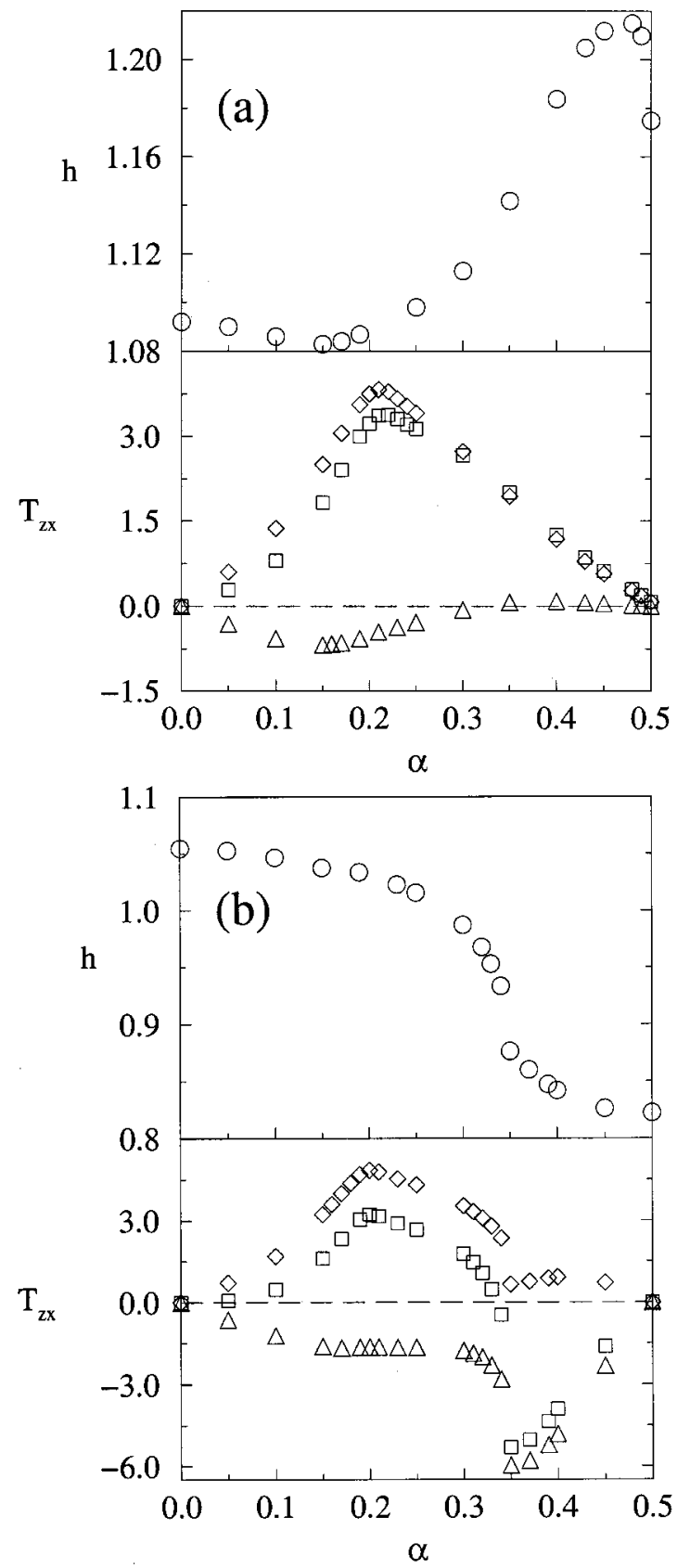

FIG. 2. (a) Shear stress $T_{z x}$ and mean separation $h$ between walls as functions of registry $\alpha$ for $\sigma_{F F} / \sigma_{W W}=1, \theta=0.0195$, and $\tau_{n}=1.0$ : film-wall component $T_{z x, F W}(\diamond)$; wall-wall component $T_{z x, W W}(\triangle)$; total $T_{z x}(\square)$. (b) Same as (a) except $\tau_{n}=5.0$.

work that must be done on the system by the surroundings to rearrange the adsorbate atoms, which do not fit as well when the walls are misaligned as they do when $\alpha=0$. On the other hand, the (negative) area under the plot of $T_{z x, W W}$ is proportional to the work done on the surroundings by the system because of the forces between the opposing walls that impel them spontaneously toward the registry $\alpha=0.5$, where, were no adsorbate present, they would fit snugly together as adjacent fcc (100) planes. Note that the maximum magnitude of this force occurs at the distance of closest approach of the walls [Fig. 2(a)].

As $\tau_{n}$ increases, the walls shift closer together on average and $T_{z x, W W}$ decreases markedly, especially in the range

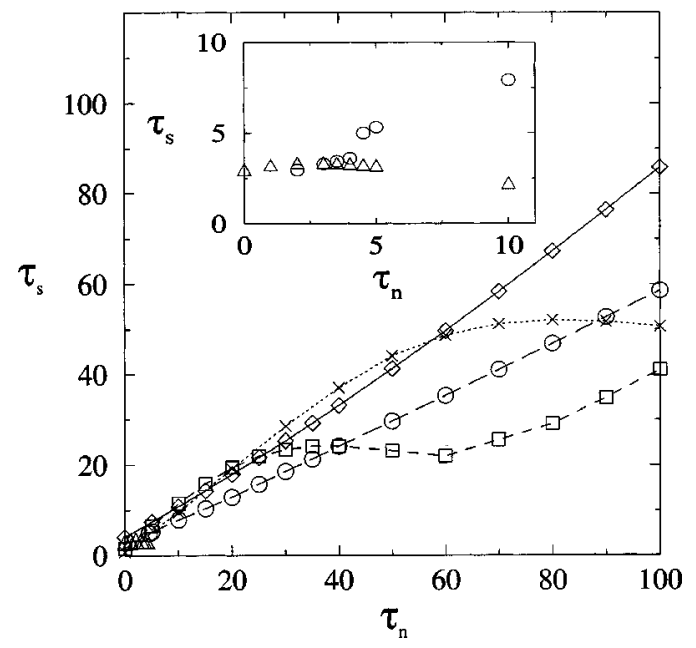

FIG. 3. Friction curves for $\sigma_{F F} / \sigma_{W W}=1$ and $\theta=0(\diamond), 0.0195(\triangle, \bigcirc)$, $0.0977(\square)$ and $0.1914(\times)$. Inset shows case $\theta=0.0195$. Open circles and triangles, respectively, refer to minima and maxima in $T_{z x}$. Lines intended merely to guide eye.

$0.3<\alpha<0.5$. Now two extrema appear in the plot of $T_{z x}$ vs $\alpha$ : a maximum determined by $T_{z x, F W}$ and a minimum determined by $T_{z x, W W}$. When $\tau_{n}$ becomes sufficiently large, the depth of the minimum exceeds the height of the maximum and the overall yield stress is determined by the former. By $\tau_{n}=5.0$ the stable registry has shifted from $\alpha=0( \pm n)$ to $\alpha=0.5( \pm n / 2, n$ odd) [Fig. 2(b) ]. It would appear that since the slope of $T_{z x}$ is positive at both integral and half-integral registries, slight displacements from either would be opposed by a net restoring force. This is so, but the integral registries are actually metastable. The free energy is minimum at the half-integral registries, as can be appreciated by observing from Fig. 2(b) that the area under the curve $T_{z x}$ is negative. In order to initiate sliding, the applied shear stress must exceed the magnitude of the minimum at $\alpha_{\min } \simeq 0.35$, which has a value $\tau_{s} \simeq 5.3$. [This point is plotted in Fig. 3 (inset) as an open circle.] The work that must be done on the system in order to carry it over the barrier to sliding, which is proportional by $\mathrm{Al}$ to the negative of the area under the plot of $T_{z x}$ from $\alpha_{\min }$ to 0.5 , is that needed to overcome the wall-wall force. The rearrangement of adsorbate in this case counts for little.

Figure 3 (inset) shows the friction curve for the case $\sigma_{F F} / \sigma_{W W}=1$ and $\theta=0.0195$. The open triangles and circles correspond, respectively, to the magnitudes of the maxima and minima in $T_{z x}$ as a function of $\alpha$. Below the "threshold" value of $\tau_{n} \simeq 3.5$ the yield stress is determined by film-wall interactions; above the threshold it is determined by wallwall interactions. Figure 3 also shows the effect of increasing coverage. As expected, the threshold rises with $\theta$. The more adsorbate atoms are present, the greater must be the load in order to bring the walls sufficiently close together that wallwall interactions become significant.

By similar reasoning we expect the threshold to increase with increasing size of the adsorbate atom at fixed coverage. This expectation is borne out by the friction curves in Fig. 4 for $\theta=0.0977$ and $\sigma_{F F} / \sigma_{W W}=0.9,1.0$, and 1.1. The larger the adsorbate atom, the higher the load needed to overcome 


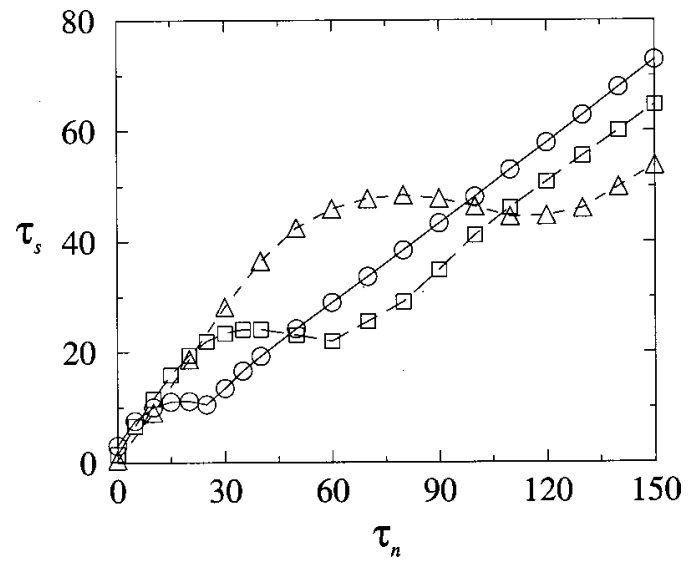

FIG. 4. Friction curves for $\theta=0.0977$ and $\sigma_{F F} / \sigma_{W W}=0.9(\bigcirc), 1.0(\square)$, and $1.1(\triangle)$.

the repulsive portion of the film-wall interactions and bring the walls close enough that wall-wall forces become nonnegligible.

For comparison we include in Fig. 3 the case of no adsorbate $(\theta=0)$, for which the competition between filmwall and wall-wall forces is absent from the beginning $\left(\tau_{n}\right.$ $=0)$. The friction curve appears linear, although scrutiny reveals a slight upward concavity. In this special case the shape of the friction curve can be rationalized by the socalled "cobblestone" model, ${ }^{10,19}$ in which the atoms of one wall are regarded as the wheels of a vehicle and those of the other wall as cobblestones in a roadway. Initially the vehicle is at rest with the wheels seated in gaps between stones. To initiate movement of the vehicle, one must apply a force $f$ parallel with the surface of the road. The component of $f$ must exceed the component of gravitational force $(w)$ parallel with the effective ramp up which the wheels must roll. Equilibrium mechanics yields the relation $f=m w$, where $m$ is the maximum slope of the ramp. This analysis implicitly assumes that all wheels are in equivalent environments (i.e., all are subject to the same effective ramp). In our model the atoms in wall 2 correspond to the wheels and the atoms in wall 1 to the cobblestones. By symmetry every atom in wall 2 is the same potential field. Thus, the relation corresponding to $f=m w$ is $F_{s, \max }=m T_{z z}$, or $\tau_{s}=m \tau_{n}$, where the last results from dividing the previous by $A$. This is strictly so only if the atoms behave as hard spheres. The actual LennardJones potentials render them "soft," so that as $\tau_{n}$ increases, the walls seat more snugly at the stable registries and the slope of the effective ramp increases. Hence, the slight concavity observed above. The near linearity of the high-load portions of the friction curves for $\theta \neq 0$ (Figs. 3 and 4) may be due to the same mechanism. The presence of adsorbate, however, seems to decrease the slope of the ramp.

\section{CONCLUSION}

To the best of our knowledge, the phenomenon we describe here has not been definitively observed in proximalprobe experiments. However, using an ultra high vacuum (UHV) tribometer, McFadden and Gellman ${ }^{20}$ measured coefficients of static friction at one load between single-crystal
$\mathrm{Cu}(111)$ faces on which were adsorbed alcohols. Unfortunately, at coverages below one monolayer, the $\mathrm{Cu}$ metal surfaces tend to "wet" each other through holes in the adsorbed film. Hence, the shear yield strength is determined by $\mathrm{Cu}-\mathrm{Cu}$ interactions. The ideal experiment would utilize an AFM under UHV, so that tip and substrate surfaces could be reproducibly prepared and the coverage precisely controlled. The tip and substrate should not wet each other, but the adsorbate should wet both tip and substrate in order to avoid "islanding" of the adsorbate.

The present results refer to fixed coverage, rather than fixed partial pressure (or chemical potential $\mu$ ) of adsorbate, whereas the latter variable is more likely to be controlled in real experiments than the former. We note, however, that if diffusion of adsorbate were relatively slow, then $\theta$ might remain fixed on the time scale of the measurement. In order to compare the predictions of our model directly with experiments in which $\mu$ is the controlled variable rather than $\theta$, we are undertaking grand isostress ensemble Monte Carlo simulations, ${ }^{21}$ in which $\mu$ replaces $N(\theta)$ as a fixed thermodynamic state variable.

\section{ACKNOWLEDGMENTS}

We thank the Office of Naval Research, the National Science Foundation, and the Petroleum Research Fund of the American Chemical Society for support. We are also grateful for the assistance of the Center for Materials Research and Analysis and the Research Computing Facility of the University of Nebraska.

${ }^{1}$ B. Bhushan, J. N. Israelachvili, and U. Landman, Nature (London) 374, 607 (1995).

${ }^{2}$ J. N. Israelachvili, P. M. McGuiggan, and A. M. Homola, Science 240, 189 (1988).

${ }^{3}$ J. van Alsten and S. Granick, Phys. Rev. Lett. 61, 2570 (1988).

${ }^{4}$ C. M. Mate, G. M. McClelland, R. Erlandsson, and S. Chiang, Phys. Rev. Lett. 59, 1942 (1987).

${ }^{5}$ Physics of Sliding Surfaces, edited by B. N. J. Persson and E. Tossati (Kluwer, Dordrecht, 1996).

${ }^{6}$ I. L. Singer, J. Vac. Sci. Technol. A 12, 2605 (1994).

${ }^{7}$ R. W. Carpick and M. Salmeron, Chem. Rev. 97, 1163 (1997).

${ }^{8}$ B. J. Briscoe and D. C. B. Evans, Proc. R. Soc. London, Ser. A 380, 389 (1982).

${ }^{9}$ M. L. Gee, P. M. McGuiggan, J. N. Israelachvili, and A. M. Homola, J. Chem. Phys. 93, 1895 (1990).

${ }^{10} \mathrm{~J}$. N. Israelachvili, in Fundamentals of Friction: Macroscopic and Microscopic Processes, edited by I. L. Singer and H. M. Pollock (Kluwer, Dordrecht, 1992).

${ }^{11}$ U. D. Schwarz, W. Alders, G. Gensterbaum, and R. Wiesendanger, Phys. Rev. B 52, 14976 (1995).

${ }^{12}$ M. R. Sørenson, K. W. Jacobsen, and P. Stolze, Phys. Rev. B 53, 2101 (1996).

${ }^{13}$ J. A. Harrison, C. T. White, R. J. Colton, and D. W. Brenner, J. Phys. Chem. 97, 6573 (1993).

${ }^{14}$ G. He, M. H. Müser, and M. O. Robbins, Science 284, 1650 (1999).

${ }^{15}$ M. Schoen, D. J. Diestler, C. L. Rhykerd, Jr., and J. H. Cushman, Science 245, 1223 (1989).

${ }^{16}$ P. A. Thompson and M. O. Robbins, Science 250, 792 (1990).

${ }^{17}$ M. Lupkowski and F. van Swol, J. Chem. Phys. 95, 1995 (1991).

${ }^{18}$ M. Schoen, D. J. Diestler, and J. H. Cushman, Phys. Rev. B 47, 5603 (1993).

${ }^{19}$ D. Tabor, in Fundamentals of Friction: Macroscopic and Microscopic Processes, edited by I. L. Singer and H. M. Pollock (Kluwer, Dordrecht, 1992).

${ }^{20}$ C. F. McFadden and A. J. Gellman, Surf. Sci. 409, 171 (1998).

${ }^{21}$ D. J. Diestler, M. Schoen, and J. H. Cushman, Science 262, 545 (1993). 